\title{
Altered Gut Microbial Metabolites in Amnestic Mild Cognitive Impairment and Alzheimer's Disease: Signals in Host-Microbe Interplay
}

\author{
Li Wu ${ }^{1}$, Yuqiu Han ${ }^{1}$, Zhipeng Zheng ${ }^{1}$, Guoping Peng ${ }^{2}$, Ping Liu ${ }^{2}$, Siqing Yue ${ }^{3}$, Shuai Zhu ${ }^{1}$, Jun Chen ${ }^{1}$, \\ Hanying $\mathrm{Lv}^{1}$, Lifang Shao ${ }^{1}$, Yan Sheng ${ }^{4}$, Yulan Wang ${ }^{5}$, Liang $\mathrm{Li}^{6}{ }^{1} \mathbb{C}$, Lanjuan $\mathrm{Li}^{1,7}$ and Baohong Wang ${ }^{1,7, *}$
}

1 State Key Laboratory for Diagnosis and Treatment of Infectious Diseases, National Clinical Research Center for Infectious Diseases, Collaborative Innovation Center for Diagnosis and Treatment of Infectious Diseases, The First Affiliated Hospital, Zhejiang University School of Medicine, Hangzhou 310003, China; cfightive@zju.edu.cn (L.W.); 11918221@zju.edu.cn (Y.H.); zpzheng@zju.edu.cn (Z.Z.); 11818164@zju.edu.cn (S.Z.); $21918242 @ z j u . e d u . c n(J . C.) ; 15168236539 @ 163 . c o m$ (H.L.); 1192038@zju.edu.cn (L.S.); ljli@zju.edu.cn (L.L.)

2 Department of Neurology, The First Affiliated Hospital, Zhejiang University School of Medicine, Hangzhou 310003, China; pgpfc@163.com (G.P.); liuping908@163.com (P.L.)

3 Key Laboratory of Microbial Technology for Industrial Pollution Control of Zhejiang Province, College of Environment, Research Center of Environmental Science, Zhejiang University of Technology, Hangzhou 310032, China; yuesiqing@126.com

4 Department of Ophthalmology, The First Affiliated Hospital, Zhejiang University School of Medicine, Hangzhou 310003, China; yansheng326@126.com

5 Singapore Phenome Center, Lee Kong Chian School of Medicine, Nanyang Technological University, Singapore 308232, Singapore; yulan.wang@ntu.edu.sg

6 Department of Chemistry, Alberta University, Edmondon, AB T6G 2G2, Canada; Liang.Li@ualberta.ca

7 Research Units of Infectious disease and Microecology, Chinese Academy of Medical Sciences, Hangzhou 310003, China

updates

Citation: Wu, L.; Han, Y.; Zheng, Z.; Peng, G.; Liu, P.; Yue, S.; Zhu, S.; Chen, J.; Lv, H.; Shao, L.; et al. Altered Gut Microbial Metabolites in Amnestic Mild Cognitive Impairment and Alzheimer's Disease: Signals in Host-Microbe Interplay. Nutrients 2021, 13, 228. https://doi.org/ $10.3390 /$ nu13010228

Received: 20 November 2020 Accepted: 12 January 2021 Published: 14 January 2021

Publisher's Note: MDPI stays neutral with regard to jurisdictional clai$\mathrm{ms}$ in published maps and institutional affiliations.

Copyright: (C) 2021 by the authors. Licensee MDPI, Basel, Switzerland. This article is an open access article distributed under the terms and conditions of the Creative Commons Attribution (CC BY) license (https:// creativecommons.org/licenses/by/ $4.0 /)$.

* Correspondence: wangbaohongzju@zju.edu.cn; Tel./Fax: +86-571-8723-6756

\begin{abstract}
Intimate metabolic host-microbiome crosstalk regulates immune, metabolic, and neuronal response in health and disease, yet remains untapped for biomarkers or intervention for disease. Our recent study identified an altered microbiome in patients with pre-onset amnestic mild cognitive impairment (aMCI) and dementia Alzheimer's disease (AD). Thus, we aimed to characterize the gut microbial metabolites among AD, aMCI, and healthy controls (HC). Here, a cohort of 77 individuals (22 aMCI, $27 \mathrm{AD}$, and $28 \mathrm{HC}$ ) was recruited. With the use of liquid-chromatography/gas chromatography mass spectrometry metabolomics profiling, we identified significant differences between AD and HC for tryptophan metabolites, short-chain fatty acids (SCFAs), and lithocholic acid, the majority of which correlated with altered microbiota and cognitive impairment. Notably, tryptophan disorders presented in aMCI and SCFAs decreased progressively from aMCI to AD. Importantly, indole-3-pyruvic acid, a metabolite from tryptophan, was identified as a signature for discrimination and prediction of $\mathrm{AD}$, and five SCFAs for pre-onset and progression of AD. This study showed fecal-based gut microbial signatures were associated with the presence and progression of $\mathrm{AD}$, providing a potential target for microbiota or dietary intervention in $\mathrm{AD}$ prevention and support for the host-microbe crosstalk signals in AD pathophysiology.
\end{abstract}

Keywords: Alzheimer's disease; fecal metabolomics; intestinal microbiota; tryptophan; short-chain fatty acids

\section{Introduction}

Alzheimer's disease (AD) is one of the leading causes of disability or death in the elderly worldwide and has become a serious health problem [1]. According to the World Alzheimer Report, more than 50 million people are living with dementia mainly caused by 
$\mathrm{AD}$ and the number of these patients will be 152 million in 2050 [2]. AD typically progresses in three stages: preclinical, mild cognitive impairment (MCI), and dementia [3]. Early detection of $\mathrm{AD}$, especially from amnestic $\mathrm{MCI}(\mathrm{aMCI})$ patients with great risk for $\mathrm{AD}$ developing [4] and decades of the pathophysiological process of $\mathrm{AD}$, is important for improving prognosis and early intervention of $\mathrm{AD}$. Through decades of research, the prevailing view of $\mathrm{AD}$ pathogenesis are deposition of $\mathrm{A} \beta$ plaques and vascular pathology $[5,6]$. However, the pathogenesis of $\mathrm{AD}$ remains unclear and no efficient medical treatment is available for $\mathrm{AD}$ [3]. To improve the outcomes of $\mathrm{AD}$, studies to understand the perturbation in other pathophysiology pathways of $\mathrm{AD}$, and to detect $\mathrm{AD}$ from high-risk populations such as those with aMCI are urgently needed.

The intestinal microbiota (IM) is considered as an 'organ', producing numerous metabolites that interact with host physiology and affect both the local intestine and distant brain functions $[7,8]$. In humans, several studies have reported the difference of IM in both American [9] and Chinese patients with $A D$ [10]. In germ-free mice, $A D$ exhibited obvious reduction of cerebral amyloid beta, highly indicating a role of IM in early pathological change of $\mathrm{AD}$ [11]. Therefore, examination of perturbation in microbial metabolites could help to reveal the microbial signatures that help in improving the prognosis or ameliorating the progression of $\mathrm{AD}$. A recent study reported that metabolomics for detecting fecal metabolites could explore the links between microbiome composition, host phenotypes, and heritable complex traits [12]. The recent metabolomics studies in AD were well conducted in blood [13], urine [14], brain [15], or cerebrospinal fluid (CSF) [16], however, no functional readout of fecal microbiota-metabolomics has been reported.

In the current study, high-resolution untargeted and targeted fecal metabolomics were performed to profile and quantify the microbial signatures in a Chinese cohort including $\mathrm{AD}$ (pre-onset aMCI and early stage $\mathrm{AD}$ ) patients and a normal cognition healthy control (HC). Furthermore, we related the differential fecal metabolites of AD to intestinal dysbiosis and cognitive impairment. This approach will enable the discovery of potential microbial biomarkers that are associated with $\mathrm{AD}$, which can be utilized for improving early detection of $\mathrm{AD}$, and facilitate a better understanding of the pathogenesis of $\mathrm{AD}$ and open up therapeutic opportunities.

\section{Materials and Methods}

\subsection{Study Subjects}

The study was conducted in accordance with the Declaration of Helsinki, and the protocol was approved by the Ethics Committee of the First Affiliated Hospital, College of Medicine, Zhejiang University (2019-251). As described previously [10], the volunteers were recruited consecutively and written informed consent was obtained. All subjects underwent a complete medical history evaluation, physical examination, neurological, and neuropsychological assessment (Mini-Mental State Examination [MMSE] and the Beijing version of the Montreal Cognitive Assessment [MoCA]), neuroimaging (magnetic resonance imaging), and clinical biochemistry examinations. AD was diagnosed according to the criteria of Diagnostic and Statistical Manual (DSM)-IV [17] and guidelines of the National Institute of Neurological and Communicative Disorders and the Stroke and Alzheimer Disease and Related Disorders Association (NINCDS-ADRDA) [18]. The aMCI was diagnosed as described previously [19]. The severity of aging dementia was evaluated by the Clinical Dementia Rating (CDR) [10] with a rating scale ranging from no dementia, to questionable dementia (MCI, CDR 0.5 ) and ultimately varying stages of dementia (AD, CDR $\geq 1$ ) [19]. The gender- and age-matched normal cognition elders were recruited as healthy controls (HC), and the majority of them were the patients' spouses, who had lived together and had been on the same diet for at least twenty years.

A cohort of 77 participants was recruited (22 aMCI, $27 \mathrm{AD}, 28 \mathrm{HC}$ ). Morning fasting serum and fecal samples were freshly collected in the baseline visit $[20,21]$. The demographic information and clinical characteristics of the cohorts are summarized in Tables 1 
and 2. The sequence dataset of the fecal microbiota can be downloaded from the National Center for Biotechnology Information (NCBI) Bioproject database (PRJNA496408).

Table 1. Demographics and clinical parameters of subjects in the Alzheimer's disease (AD), amnestic mild cognitive impairment (aMCI), and normal cognition healthy control (HC) groups.

\begin{tabular}{cccc}
\hline Characteristics & HC & aMCI & AD \\
\cline { 2 - 4 } & $\mathbf{( n = 2 8 )}$ & $\mathbf{( n = 2 2 )}$ & $\mathbf{( n = 2 7 )}$ \\
\hline Age (years) & $74.25 \pm 9.03$ & $70.00 \pm 11.33$ & $74.15 \pm 11.16$ \\
Sex (male/female) & $14 / 14$ & $9 / 13$ & $15 / 12$ \\
Education (years) & $9(6-12)$ & $9(9-12)$ & $9(6-9)$ \\
BMI $\left(\mathrm{kg} / \mathrm{m}^{2}\right)$ & $22.29 \pm 2.18$ & $22.68 \pm 2.17$ & $21.87 \pm 1.15$ \\
Diabetes $(\%)$ & $0(0 \%)$ & $2(9.1 \%)$ & $5(18.7 \%)$ \\
Hypertension $(\%)$ & $9(32.1 \%)$ & $9(40.9 \%)$ & $11(40.7 \%)$ \\
Fasting glucose & $5.23 \pm 0.69$ & $6.37 \pm 1.93$ & $5.02 \pm 0.54$ \\
(mmol/L) & $138.42 \pm 17.85$ & $142.19 \pm 11.23$ & $136.79 \pm 11.38$ \\
Hemoglobin (g/L) & $11.08 \pm 5.16$ & $8.97 \pm 2.90$ & $8.75 \pm 3.66$ \\
Folic acid (ng/mL) & $484.00(435.50-716.00)$ & $510.00(379.00-685.00)$ & $383.50(341.00-506.25)$ \\
Vitamin B12 (pg/mL) & $107.05 \pm 22.31$ & $108.44 \pm 19.74$ & $103.50 \pm 35.06$ \\
TT4 (nmol/L) & $1.53(1.43-1.92)$ & $1.72(1.44-1.89)$ & $1.55(1.38-1.75)$ \\
TT3 (nmol/L) & $29.00(26.00-29.50)$ & $27.00(26.00-28.00)$ & $18.00(13.50-23.00)$ \\
MMSE & & & $* * * \# \#$ \\
MoCA & $26.00(24.50-27.00)$ & $22.00(18.00-24.00) *$ & $17.00(14.50-19.00)$ \\
& & & $* * * \#$ \\
\hline
\end{tabular}

Note: Data are given as the mean \pm SD or median (IQR). ${ }^{*} p<0.05 ;{ }^{* * *} p<0.001$ compared with HC and ${ }^{*} p<0.05$; $\# \#$ \# 0.001 compared with aMCI group. Abbreviations: HC, normal cognition healthy control; aMCI, amnestic mild cognitive impairment; AD, Alzheimer's disease; BMI, body mass index; TT3, total triiodothyronine; TT4, total thyroxine; MMSE, Mini-Mental State Examination; MoCA, Montreal Cognitive Assessment; SD, standard deviations; IQR, interquartile range.

Table 2. Sub item Montreal Cognitive Assessment (MoCA) scores of AD, aMCI, and HC groups.

\begin{tabular}{cccc}
\hline MoCA Sub Item & HC & aMCI & AD \\
\cline { 2 - 4 } & $\mathbf{( n = 2 8 )}$ & $\mathbf{( n = 2 2 )}$ & $\mathbf{( n = 2 7 )}$ \\
\hline Visuospatial & $5(4.75-5)$ & $4(3-5)$ & $3(1.25-3.75)^{* * * \#}$ \\
Naming & $3(3-3)$ & $3(2-3)$ & $2(2-3)^{* *}$ \\
Attention & $3(3-3)$ & $3(2-3)$ & $1.5(1-2.75)^{* * \#}$ \\
Language & $2.5(2-3)$ & $2(1-2)$ & $1(0-1)^{* * * \#}$ \\
Abstraction & $1.5(1-2)$ & $0(0-1)$ & $0(0-0)^{* * *}$ \\
Delayed recall & $3.5(3-5)$ & $1(0-3)^{* *}$ & $0.5(0-1)^{* * *}$ \\
Orientation & $6(5.75-6)$ & $6(5-6)$ & $3(3-5.75)^{* * \#}$ \\
\hline
\end{tabular}

Note: Data are given as the median (IQR). ${ }^{* *} p<0.01 ;{ }^{* * *} p<0.001$ compared with HC and ${ }^{\#} p<0.05$; ${ }^{\# \#} p<0.01$ compared with aMCI group. Abbreviations: $\mathrm{HC}$, normal cognition healthy control; aMCI, amnestic mild cognitive impairment; AD, Alzheimer's disease; MoCA, Montreal Cognitive Assessment; IQR, interquartile range.

\subsection{Fecal Metabolomics Analysis}

\subsubsection{Sample Preparation}

The preprocessed pipeline of fecal samples was established. First, the samples were mixed with methanol $(3 \mathrm{~mL} / \mathrm{g})$ and homogenized $(8 \mathrm{~m} / \mathrm{s}, 15 \mathrm{~s})$ with ceramic beads $(1 \mathrm{~mm})$ (Omni International, Bedford, NH, USA) [22]. Then, the supernatants after centrifugation were filtered using syringe filters $(0.22 \mu \mathrm{m}$, Millipore Corp, Billerica, MA, USA). To monitor potential contamination of sample processing, a procedural blank sample of water (Thermo Fisher Scientific, Waltham, MA, USA) was used. Quality control (QC) samples were prepared by mixing equal volumes of all fecal extraction, and processed with the real samples simultaneously. 


\subsubsection{Fecal Untargeted Metabolomics Profiling}

The metabolomics profiling was performed using a Dionex UltiMate 3000 RS ultraperformance liquid chromatography (UPLC) system coupled with Orbitrap Q Exactive mass spectrometry (MS) with a Hypersil Gold C-18 column $(2.1 \times 100 \mathrm{~mm}, 1.9 \mu \mathrm{m})$ (Thermo Fisher Scientific, Waltham, MA, USA). Both ESI+ and ESI- ionization modes were executed with a $m / z$ range $70-1050$ and a full MS scan at 60,000 resolution.

\subsubsection{Multivariate Data Analysis}

Raw data were acquired by Xcalibur 4.1 software and processed by Compound Discoverer 3.1 software (Thermo Fisher Scientific, Waltham, MA, USA) for peak detection, peak alignment, and peak integration. The processing procedures were modified from a previous method [23]. The multivariate statistical analysis including principle component analysis (PCA) and partial least-squares-latent structure discriminate analysis (PLS-DA) were performed in SIMCA-P 13.0 (Umetrics AB, Umea, Vasterbotten, Sweden) [24].

The biologically metabolites were picked out using Compound Discoverer 3.1. Briefly, the local library and online database including ChemSpider and mzCloud, of which the Human Metabolome Database (HMDB), Kyoto Encyclopedia of Genes and Genomes (KEGG), and Biocyc were used. The identity of the candidate biomarkers were confirmed by the standard compound libraries of both our lab and the public database.

\subsection{Targeted Profiling of Fecal Tryptophan Metabolites}

The mass list of endogenous tryptophan metabolites (Table S1) was compiled using the "Search Mass Lists" node of the Compound Discoverer 3.1, conducting the full-MS scan to pick out the tryptophan metabolites from samples.

\subsection{Targeted Profiling of Fecal Short-Chain Fatty Acids (SCFAs)}

Short-chain fatty acid (SCFA) profiles and quantification were performed by gas chromatography (GC)-MS based on an Agilent 7890B with a single quadrupole MS (5977, Agilent Technologies, Santa Clara, CA, USA). The samples were prepared by mixing with $10 \%$ isobutanol, and homogenized twice $(50 \mathrm{HZ}, 30 \mathrm{~s})$ using tissuelyser (QIAGEN, Hilden, Germany) and centrifuged (12,000 rpm, $5 \mathrm{~min}$ ). Then, the chloroform derivatization process was modified based on that previously described $[25,26]$. The identity of SCFAs were based on the retention time and fragments compared with the chemical standards (Table S2) and quantified by external standard calibration methods using Mass Hunter software (Agilent Technologies, Santa Clara, CA, USA) [27].

\subsection{Targeted Profiling of Fecal Bile Acids}

The bile acids (BAs) were determined using an UPLC tandem MS system (6470, Agilent Technologies, Santa Clara, CA, USA). As previously described [28,29], the samples were extracted by methanol solvent containing an internal standard (Table S3), processed with rapid freeze-thaw cycles, centrifuged, and finally filtered $(0.22 \mu \mathrm{m}$ filter $)$. The raw data were processed using Mass Hunter software. The BAs were identified by referring to the retention time and ion pairs of standards (Tables S4 and S5) and quantified by the internal standard calibration curves.

\subsection{Measurement of Circulating Lipopolysaccharide Level}

The serum lipopolysaccharide (LPS) levels were measured using the limulus amoebocyte lysate (LAL) chromogenic endpoint assay (Hycult Biotech, Uden, The Netherlands) [30] by the spectrophotometer (Biotek, Winooski, VT, USA) with the absorbance at $405 \mathrm{~nm}$.

\subsection{Statistical Analysis}

The univariate analysis of each metabolite was performed by one-way ANOVA or Kruskal-Wallis test using SPSS software package (version 16.0, SPSS, Inc., Chicago, IL, 
USA) and GraphPad Prism 6 (GraphPad software, La Jolla, CA, USA). A $p$ value $<0.05$ was considered statistically significance. Multiple comparison corrections were conducted using False Discovery Rate (FDR) correction for adjusted $p$ value (or q value). Correlation analysis was performed using Spearman rank correlation analysis. A hierarchical clustering heat map was established by correlation coefficient (Cluster 3.0 software) [31]. The binary logistic regression models were built for biomarkers based on the backward-selected metabolites. The area under the receiver operating characteristic (ROC) curves (AUC) were used to assess the predictive ability of models $[10,32]$. The schematic workflow of statistical analysis is listed in Figure S1.

The detailed information of all methods and materials are provided in the Supplementary Materials.

\section{Results}

\subsection{Distinguishing Metabolomics Profiles among $A D, a M C I$, and $H C$}

The fecal metabolomics profiles of patients with pre-onset aMCI and AD were clearly separated from HC individuals in the PCA score plot, and even showed the separation trend between $\mathrm{aMCI}$ and $\mathrm{AD}$ groups (Figure 1a). Furthermore, based on the top twenty-five differential metabolites, the PLS-DA plot presented the separation among the AD, aMCI, and $\mathrm{HC}$ groups (Figure $1 \mathrm{~b}$ ). Interestingly, the altered metabolites in AD mainly pointed to the disturbance of tryptophan metabolism (Table S6).

(a)

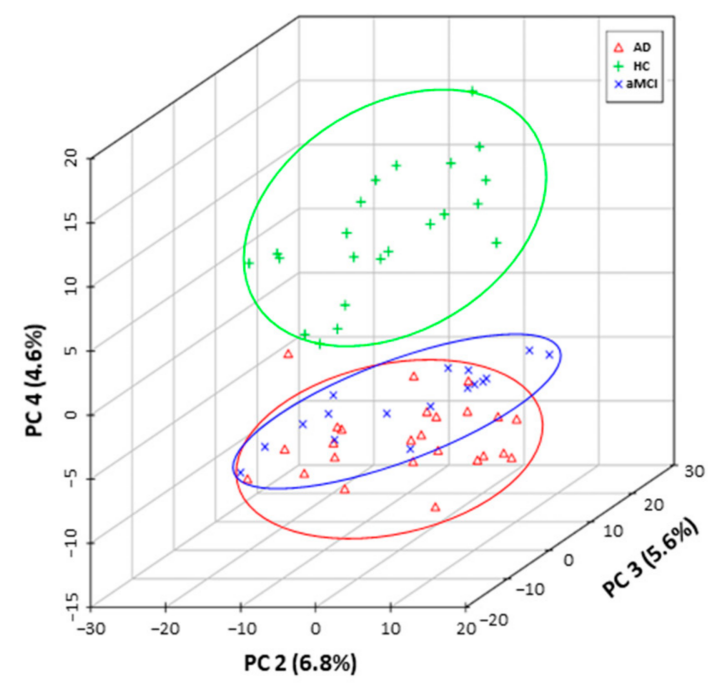

(b)

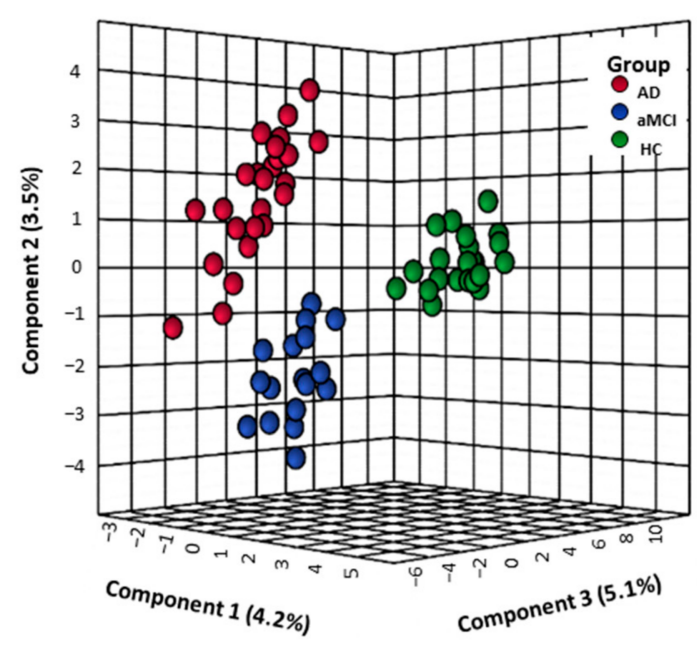

Figure 1. Fecal metabolomics profiles separated AD patients from HC. Differential fecal metabolomics profiles among aMCI $(n=17), \operatorname{AD}(n=25)$ and HC ( $n=22)$ were presented by (a) PCA score plot based on untargeted high-throughput UHPLC-orbitrap Q Exactive-MS metabolic profiling and (b) PLS-DA model based on top twenty-five differential metabolites. Abbreviations: HC, normal cognition healthy control; aMCI, amnestic mild cognitive impairment; AD, Alzheimer's disease; PCA, principle component analysis; PLS-DA, partial least-squares-latent structure discriminate analysis; UHPLC, ultra-high performance liquid chromatography; MS, mass spectrometry.

\subsection{Disturbed Metabolic Pathways of Tryptophan in Patients with $A D$}

We profiled all metabolites of the tryptophan pathways by setting an in-house mass list library based on KEGG data. As shown in Figure 2a-d, in total, nine tryptophan metabolites were detected and quantified. Metabolites were annotated to the three reported pathways, two in the host (the kynurenine pathway and serotonin pathway), and one in the intestine (indole derivatives pathway). We did not find changes in the kynurenine and kynurenic acid in patients with AD (Figure 2a,d), indicating that tryptophan metabolized by the host was not altered in AD. Interestingly, the findings showed that the metabolites 
in the serotonin pathway significantly decreased in AD (Figure 2b,d). For example, 5hydroxytryptophan (5-HTP), a precursor of 5-HT, was significantly decreased in AD compared with HC $(p=0.022)$, and showed a reduced trend in aMCI when compared with HC. Additionally, the 5-HT family member, DL-5-methoxytryptophan (DL-5-MTP), was markedly depleted in both aMCI and AD compared with HC $(p<0.001)$. These findings indicate that the tryptophan co-metabolized by host and microbiota was significantly disturbed in AD.

(a)

(b)

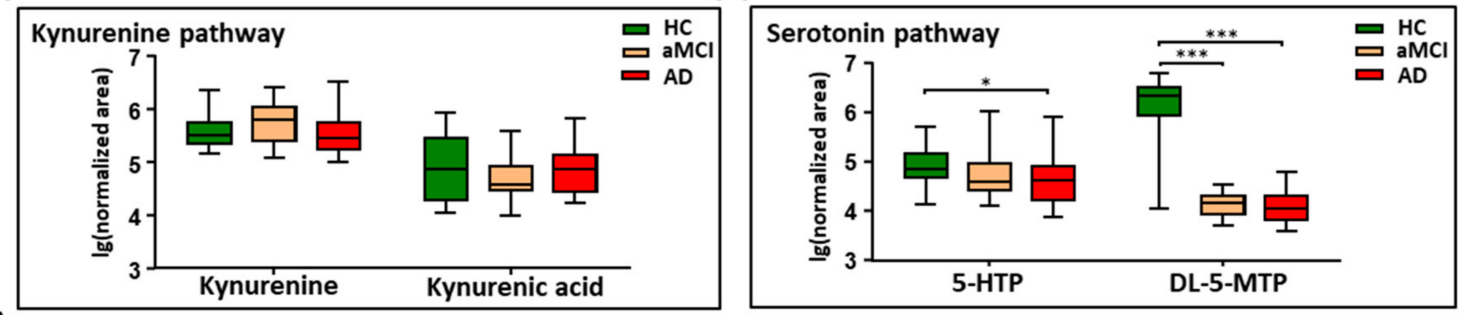

(c)

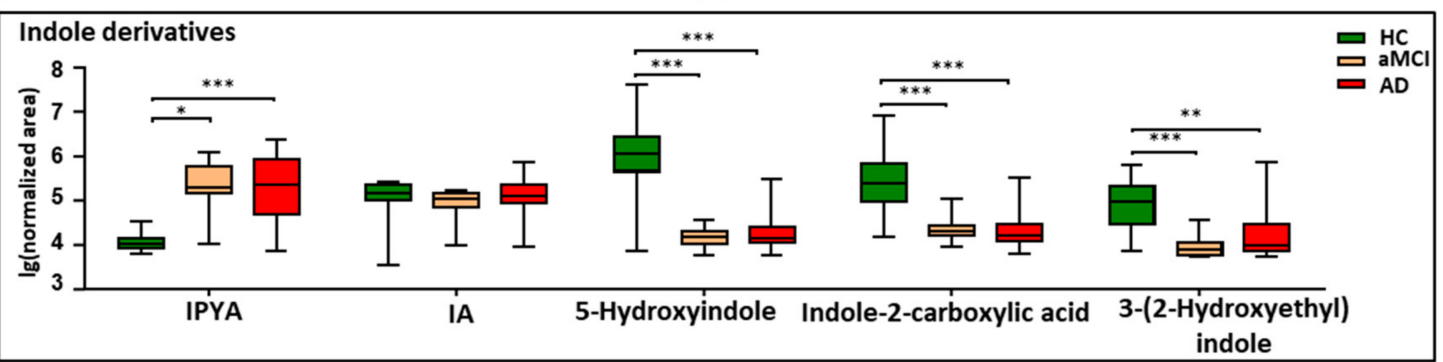

(d)

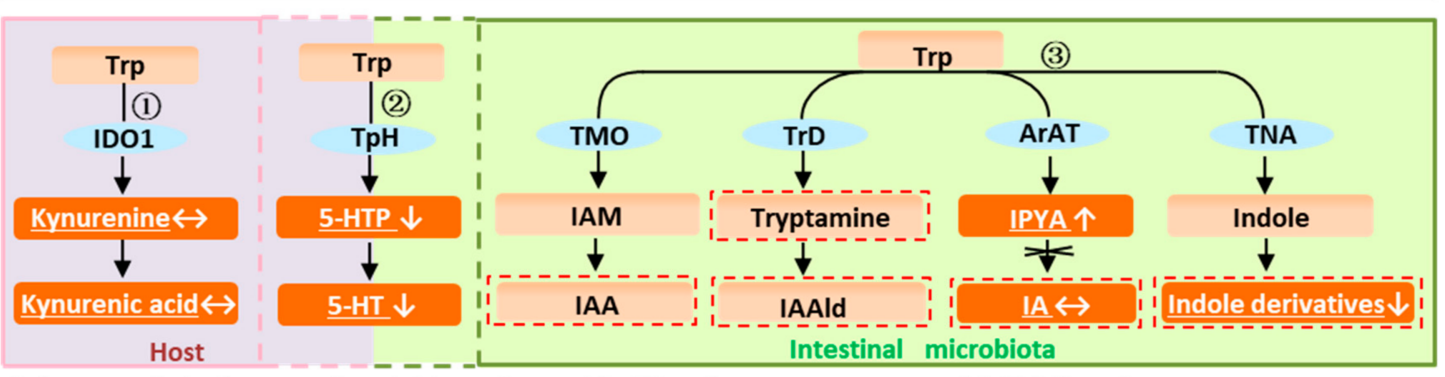

$\uparrow$, increased; $\downarrow$, decreased, and $\leftrightarrow$ unchanged in AD patients;

Figure 2. Dysregulation of fecal tryptophan metabolic pathway in AD patients. (a-c) Comparison of detected tryptophan metabolites in the kynurenine pathway, serotonin pathway, and indole derivatives among AD, aMCI, and HC groups. (d) There are three major tryptophan metabolism pathways: (1) Kynurenine pathway mainly occurs in the liver or extrahepatic organs; (2) Although neurotransmitter serotonin (5-hydroxytryptamine [5-HT]) can be synthesized in the brain, $90 \%$ of the body's 5-HT is produced in the gut; (3) Tryptophan was directly transformed into indole and its derivatives in the gut controlled by gut microbiota, and many of the indole derivatives are potential AhR ligands. The detected tryptophan metabolites in the current study were marked with a white color and underlined. Note: Data are given as median with range. $p$ values were determined by one-way ANOVA or Kruskal-Wallis test. ${ }^{*} p<0.05 ;{ }^{* *} p<0.01 ;{ }^{* * *} p<0.001$. Abbreviations: Trp, tryptophan; IDO1, indoleamine 2,3-dioxygenase 1; TpH, tryptophan hydroxylase; TMO, tryptophan 2-monooxygenase; TrD, tryptophan decarboxylase; ArAT, aromatic amino acid aminotransferases; TNA, tryptophanase; 5-HTP, 5-hydroxytryptophan; DL-5-MTP, DL-5-methoxytryptophan; IAM, indole-3-acetamide; IAA, indole acetic acid; IAAld, indole-3-acetaldehyde; IPYA, indole-3-pyruvic acid; IA, indoleacrylic acid; AhR, aryl hydrocarbon receptor.

Furthermore, we revealed that indole derivatives of the tryptophan pathway metabolized by IM were markedly disturbed (Figure 2c,d). For instance, the derivative indole-3-pyruvic acid (IPYA) was progressively enriched from aMCI and AD compared with HC $(p<0.05)$. Of note, the indole derivatives, acting as the aryl hydrocarbon receptor $(\mathrm{AhR})$ ligands, were markedly depleted in both $\mathrm{aMCI}$ and $\mathrm{AD}$ when compared with $\mathrm{HC}$ $(p<0.01)$. These findings show that the tryptophan pathways metabolized by IM were significantly disturbed in $\mathrm{AD}$, especially the AhR ligands of indole derivatives. After 
adjusting the significance level for multiple comparisons, these tryptophan metabolites in $\mathrm{aMCI}$ and $\mathrm{AD}$ patients were still significantly different from HC $(p<0.001)$ (Table S6).

\subsection{Fecal SCFAs Associated with Progression from $\mathrm{HC}$ to $a M C I$ and $A D$}

The targeted metabolomics profiling of SCFAs based on GC-MS was performed, which were the key gut-derived metabolites. In total, eight SCFAs were detected in the feces of all study subjects (Figure S2). Compared with HC, seven SCFAs showed a progressively decreased trend from aMCI to $\mathrm{AD}$, six of the seven SCFAs were significantly lower in both aMCI and $\mathrm{AD}(p<0.05)$, and the valeric acid was markedly decreased in $\mathrm{AD}$ (emphp < 0.05) (Figure 3a). Interestingly, when adjusting the observed significance level for multiple comparisons, five SCFAs including formic acid, acetic acid, propanoic acid, 2-methylbutyric acid, and isovaleric acid showed a significant difference between aMCI and AD patients $(p<0.05)$ (Table S7). Together, these results show that fecal SCFAs were changed associated with the disease progression of $\mathrm{AD}$.

(a)

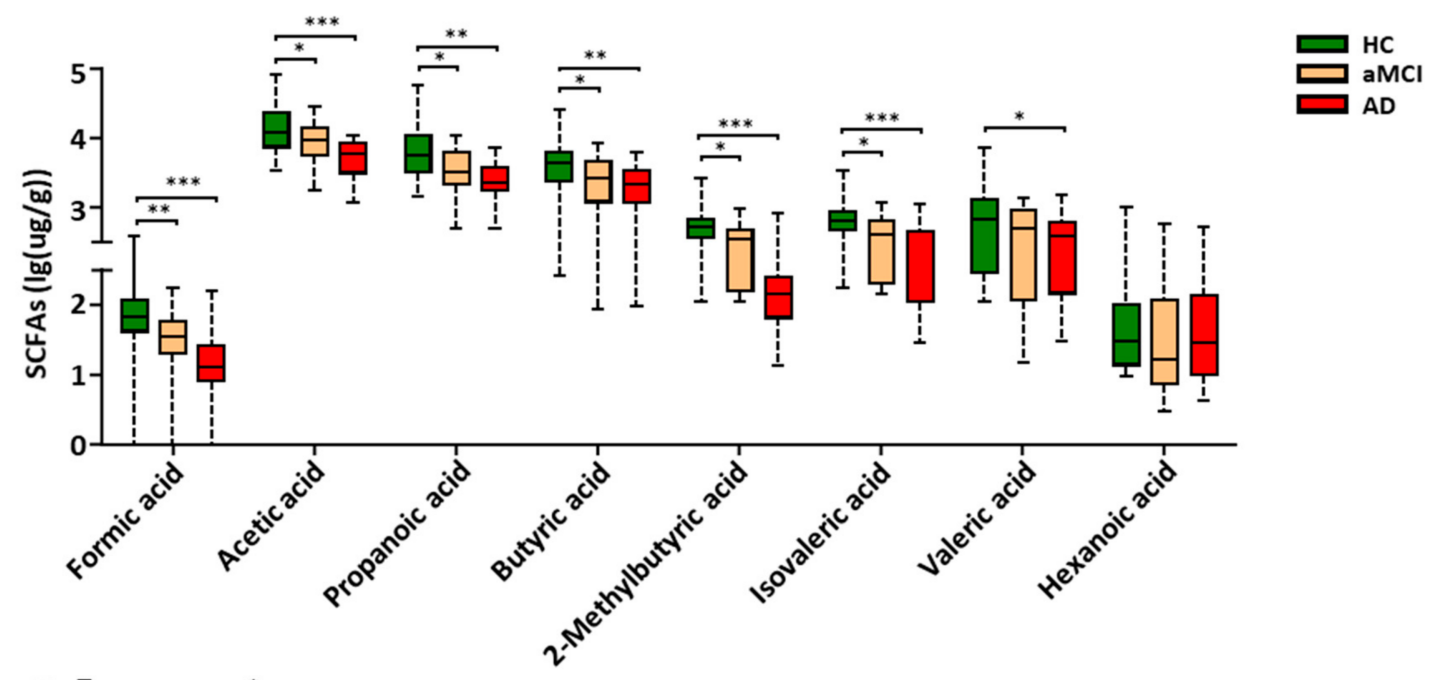

(b)

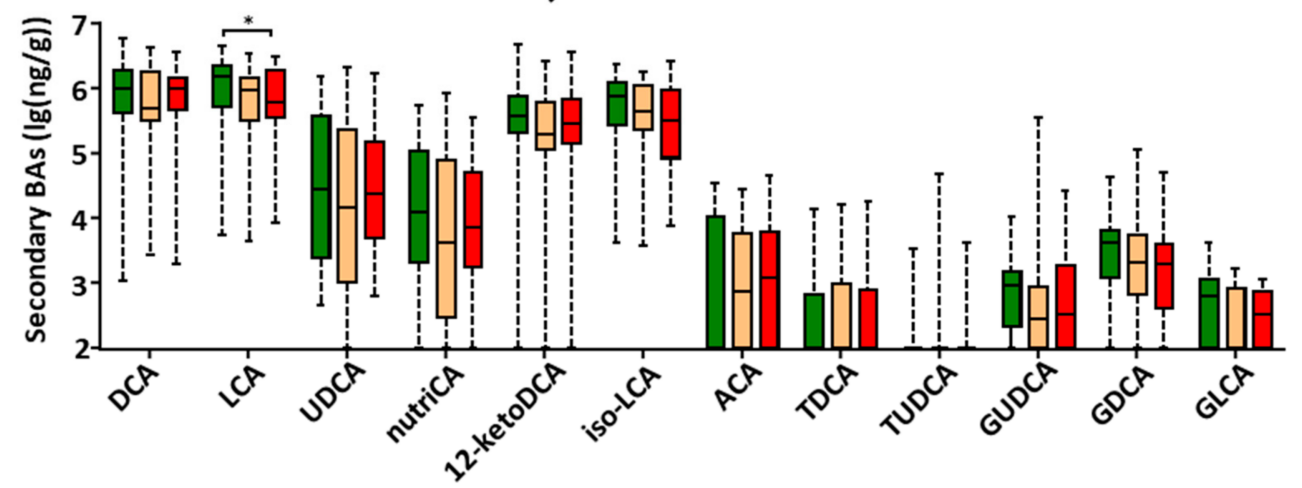

Figure 3. Progressively decreased SCFAs and LCA from HC to aMCI and AD patients. (a,b) Comparison of fecal SCFAs and fecal secondary bile acids among the $\mathrm{HC}$, aMCI, and AD groups. Note: Data are given as median with range and $P$ values were calculated using one-way ANOVA or Kruskal-Wallis test. ${ }^{*} p<0.05 ;{ }^{* *} p<0.01$; ${ }^{* * *} p<0.001$. Abbreviations: SCFAs, short-chain fatty acids; DCA, deoxycholic acid; LCA, lithocholic acid; UDCA, ursodeoxycholic acid; nutriCA, nutriacholic acid; 12-ketoDCA, 12-ketodeoxycholic acid; iso-LCA, isolithocholic acid; ACA, allocholic acid; TDCA, taurodeoxycholic acid; TUDCA, tauroursodeoxycholic acid; GUDCA, glycoursodeoxycholic acid; GDCA, glycodeoxycholic acid; GLCA, glycolithocholic acid; BAs, bile acids; ANOVA, analysis of variance.

\subsection{Perturbation of Fecal Bile Acids in AD Patients}

Due to the low relative abundance of fecal BAs, the targeted metabolomics were conducted to examine the BAs metabolism. A total of thirty fecal BAs were profiled and quantitatively examined (Figure S3). Here, a total of eighteen primary (Figure S4a) 
and secondary BAs (Figure 3b) were detected among all subjects of the cohort. Of note, only the cytotoxic lithocholic acid (LCA) [33-35] showed a gradually decreased trend from $\mathrm{HC}$ to $\mathrm{aMCI}$ and $\mathrm{AD}$, and a significant reduction in $\mathrm{AD}$ when compared with $\mathrm{HC}$ subjects ( $p=0.039$ ) (Figure $3 b$ ), while the multiple comparison correction results showed no significant difference in LCA between $\mathrm{AD}$ and HC $(p=0.126)$. There was no difference in the ratio of lithocholicacid (LCA) to chenodeoxycholic acid (CDCA), total LCA to total CDCA, total primary BAs, total secondary BAs and the ratio of total secondary to primary BAs in AD (Figure S4b-e).

\subsection{Association between the Disturbed Microbes of AD and Fecal Metabolites}

The Spearman correlation analysis was used to analyze the association between disordered microbes (Table S8) and the perturbed microbial signatures of AD including metabolites of the tryptophan pathway, SCFAs, and LCA. A significant linkage between the altered microbiota and metabolic perturbation of AD was presented (Figure 4). Many of the identified compounds are well known gut microbial metabolites such as DL-5MTP, IPYA, LCA, and SCFAs. Some microbial members only revealed a single metabolite association, for example, the connection between Lachnospiraceae and 2-methylbutyric acid and some members had multiple connectivities, for example, Clostridia was statistically linked with the presence of three fecal metabolites including DL-5-MTP, formic acid, and 2-methylbutyric acid.
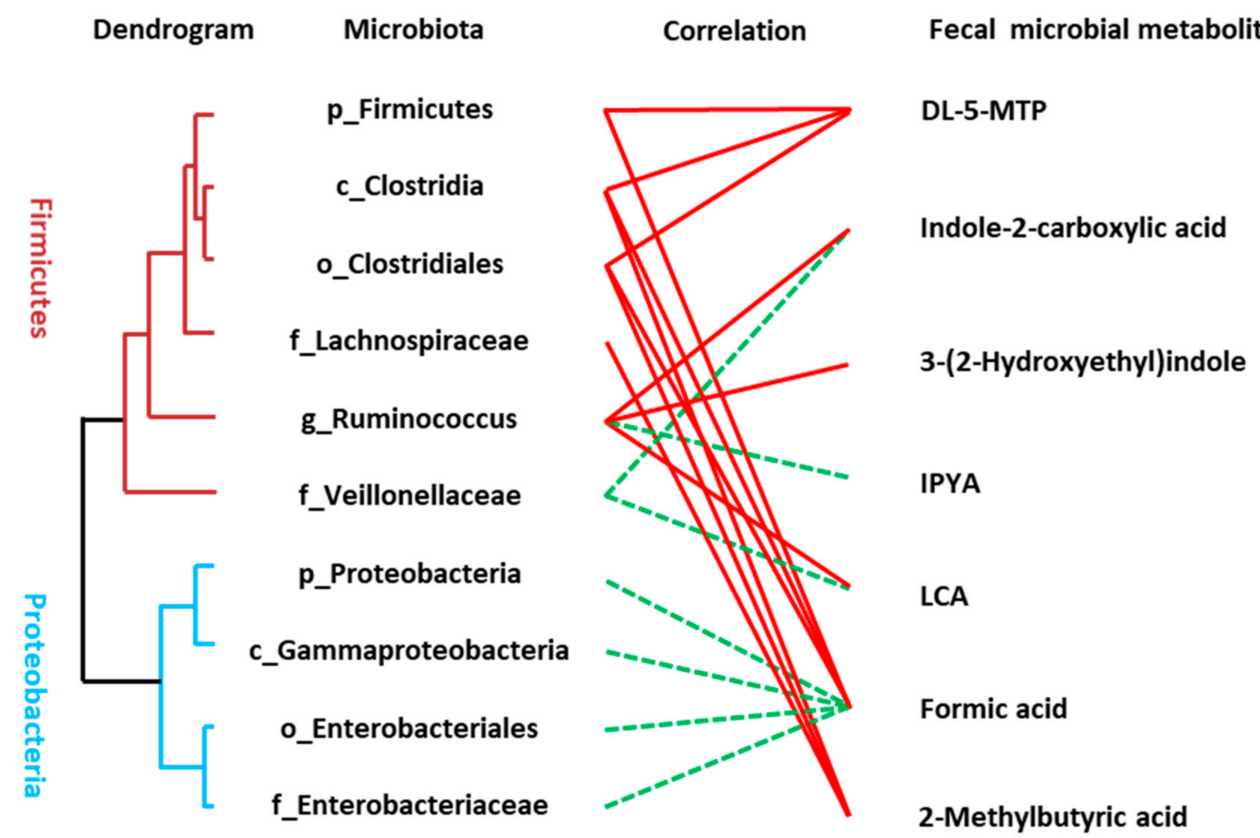

Figure 4. Linkage between altered microbes and differential microbial metabolites of AD patients. Correlation analysis between the altered microbiota and fecal metabolites were conducted using Spearman rank correlation or Pearson correlation analysis (red line means significantly positive correlation and green line means significantly negative correlation). The hierarchical cluster of gut microbiota was achieved based on the correlation coefficient of the relative abundance.

Due to the remarkably altered concentration of SCFAs in feces of AD patients, we compared the reported 15 SCFA-producing bacteria [36-38] between HC, aMCI, and AD patients. As shown in Figure S5, Clostridia, with the capability of producing SCFAs like acetate, proprionate, butyratem and its phylum Firmicutes as well as the Clostridiales, $R u$ minococcaceae, Ruminoсоссиs at order, family and genus levels, were significantly decreased in $\mathrm{AD}$ patients compared with aMCI or $\mathrm{HC}$, while the propionate-producing Bacteroides was increased in aMCI patients compared with $\mathrm{AD}$ and $\mathrm{HC}$ (Figure S5). The consistent results of SCFA-producing bacteria and fecal SCFA concentration suggested that the de- 
creased clusters of bacteria in Firmicutes, Clostridia, and Clostridiales of AD patients might contribute to the reduction of SCFAs.

\subsection{Serum Levels of Endotoxin Prone to Increase in $A D$}

Given the role of pro-inflammatory lipopolysaccharide (LPS) in cognitive impairment [39], the circulating LPS level was measured. There was an increasing trend in the serum LPS of pre-onset aMCI (aMCI vs. HC, $p=0.210$ ) and AD (AD vs. HC, $p=0.557$ ) patients compared with HC individuals (Figure S6). These results exhibited a prone "leaky gut" in patients with both aMCI and AD.

\subsection{Association between the Altered Metabolites of $A D$ and Cognitive Impairment}

The association between the fecal altered microbial signatures and parameters reflecting disease severity (CDR), cognitive function (MMSE and MoCA) and almost all the subdomains were shown (Figure $5 a, b)$. For example, the increased cognitive impairment (MoCA) positively correlated with the reduced serotonin (5-HTP) and SCFAs, while was negatively correlated to the increased indole-3-pyruvic acid of $\mathrm{AD}(p<0.05)$. These decreased SCFAs were negatively associated with increased CDR $(p<0.05)$. Indole derivatives (5-hydroxyindole and indole-2-carboxylic acid) and SCFAs (including 2-methylbutyric acid and isovaleric acid) were positively associated with cognitive impairment (MMSE) $(p<0.05)$. Furthermore, a significant correlation existed between these fecal microbial signatures of $\mathrm{AD}$ and the cognitive impairment (almost all the subdomains of MoCA and MMSE) (Figure $5 b$ ). These results showed that the perturbed microbial signatures of $A D$ correlated with MoCA and MMSE items of cognitive impairment.

(a)

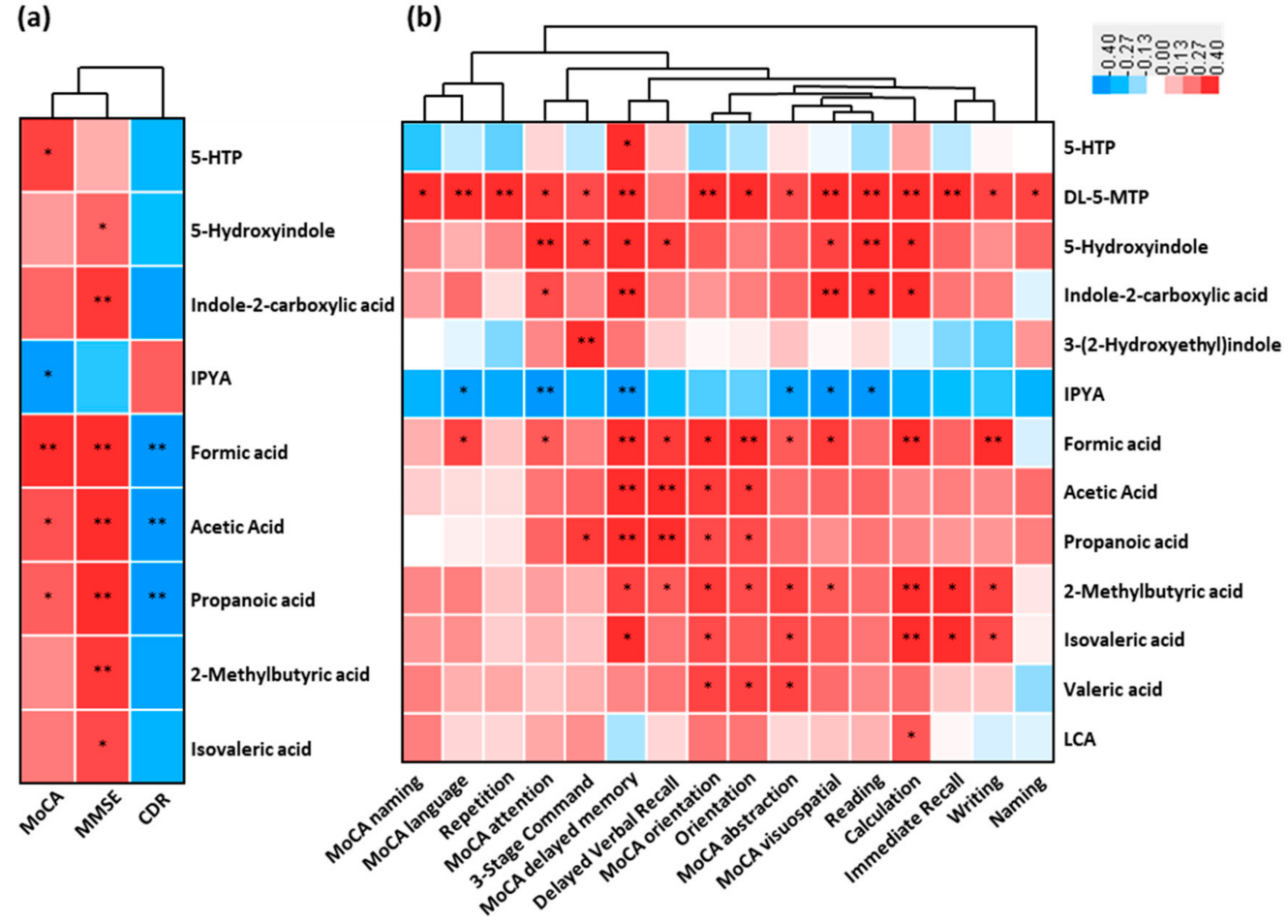

Figure 5. Linkage between altered metabolites of AD and cognitive impairment. Heat maps of Spearman rank correlation between the altered fecal metabolites including tryptophan metabolites, SCFAs, and bile acids, and (a) clinical parameters reflecting cognition function and disease severity, and (b) sub items in MoCA (naming, language, attention, delayed memory, orientation, abstraction and visuospatial) and MMSE sub domains (repetition, 3-stage command, delayed verbal recall, orientation, reading, calculation, immediate recall, writing and naming). Note: ${ }^{*} p<0.05 ;{ }^{* *} p<0.01$. Red means positive correlation and blue means negative. Abbreviations: MoCA, Montreal Cognitive Assessment; MMSE, Mini-Mental State Examination; CDR, Clinical Dementia Rating. 


\subsection{Classification of AD from aMCI and HC by Fecal Microbial Signatures}

Among the fourteen fecal differentiating microbial metabolites of $\mathrm{AD}$, a panel of six biomarkers including IPYA and five SCFAs effectively discriminated aMCI and AD from HC based on ROC curve analysis (aMCI vs. HC, AUC $=0.999, p=0.000$; AD vs. HC, $\mathrm{AUC}=0.999, p=0.000$ ) (Figure 6a,b). Notably, IPYA accurately classified aMCI $(\mathrm{AUC}=0.955, p=0.000)$ and $\mathrm{AD}(\mathrm{AUC}=0.958, p=0.000)$ from HC (Figure 6a,b; Table S9). Additionally, five SCFAs discriminated aMCI from AD (AUC $=0.922, p=0.000)$ and $\mathrm{HC}$ (AUC $=0.848, p=0.000)$ as well as AD from HC (AUC $=0.953, p=0.000)$ (Figure 6a-c).

(a)

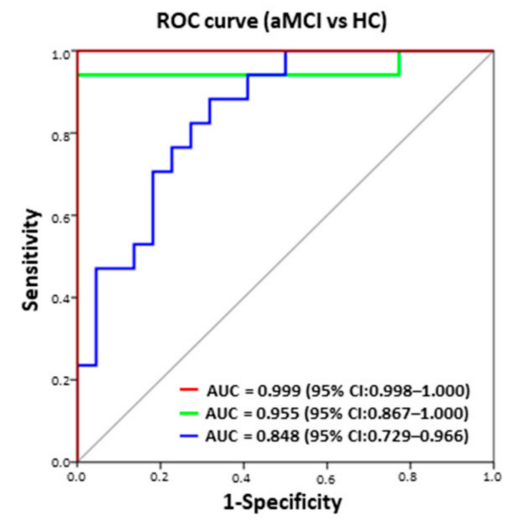

(b)

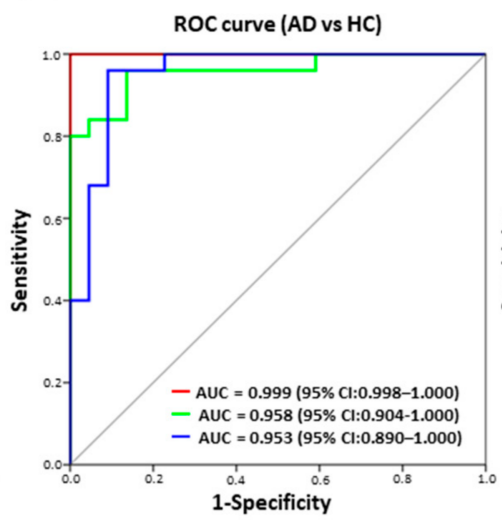

(c)
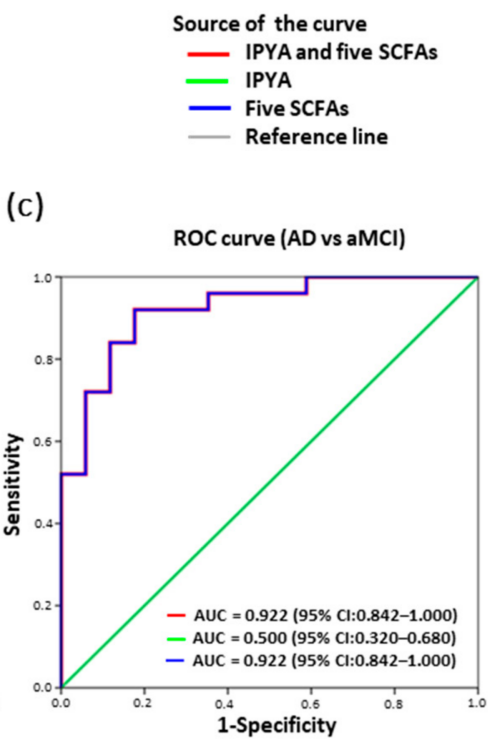

Figure 6. Classification and prediction of AD patients using the panel of six fecal microbial metabolites. Predictive models based on IPYA of tryptophan metabolites and five SCFAs (formic acid, acetic acid, propanoic acid, 2-methylbutyric acid and isovaleric acid) were built by performing binary multivariable logistic regression analysis. ROC curves were calculated for discriminating (a) aMCI from $\mathrm{HC}$, (b) AD from $\mathrm{HC}$, and (c) AD from aMCI. Abbreviations: ROC, receiver operating characteristic; AUC, area under the ROC curve; CI, confidence interval.

\section{Discussion}

Previous studies were focused on serum, CSF, and tissue metabolomics profiles of $\mathrm{AD}$ progression. However, our previous study found a great contribution of IM in AD progression and no fecal metabolomics profiles had been previously investigated. Thus, studies to explore whether fecal microbiota-metabolomics signatures could serve as new noninvasive biomarkers to differentiate $\mathrm{AD}$ patients in crucial stages including pre-onset $\mathrm{aMCI}$ and early $\mathrm{AD}$ dementia can greatly benefit early $\mathrm{AD}$ detection and intervention. By integrating high-throughput UPLC/MS and GC/MS-based metabolomics studies of fecal metabolites, the fecal metabolic dysregulation of $\mathrm{AD}$ pointed to tryptophan, SCFAs, and BAs pathways, which linked with gut dysbiosis and cognitive impairment. Moreover, the study showed that six microbial signatures, especially IPYA, could accurately classify both aMCI and AD from HC. Our study presented the fecal microbial signatures of AD, providing novel biomarkers and a therapeutic strategy for $\mathrm{AD}$ progression.

The IM is a crucial player in human physiology. The dialogue between the microbiota and host are mediated by metabolites that are either produced by the microbiota or derived from the transformation of environmental or host molecules $[7,8]$. Here, we found that the distinct fecal metabolomics of $\mathrm{AD}$ including pre-onset stage $\mathrm{aMCI}$ and $\mathrm{AD}$ dementia compared with the cognitively healthy status. The perturbation in three categories of metabolites including tryptophan, SCFAs, and bile acids were found in AD patients. These metabolites have been recognized as the essential array at the interface between microorganisms and the host [7]. It is widely accepted that tryptophan can serve as a 
key prerequisite that coordinates the gastrointestinal physiology and CNS function [7], which was achieved through regulation of indole derivatives, the kynurenine pathway, and serotonin synthesis [7]. Here, we found that the fecal metabolomics of AD were characterized with the significant perturbation of tryptophan metabolism, especially the indole derivatives and serotonin synthesis, and were significantly linked with intestinal dysbiosis and AD development. The serotonin in both enteric and CNS is a key element in the gut-brain axis, acting as a neurotransmitter [40]. The abnormal 5-HT synthesis caused by intestinal dysbiosis could affect the pathological process of AD [41]. In addition, the wellknown AhR signaling of tryptophan metabolites constituting the interface of microbiotagut-brain axes [42], could suppress pro-inflammatory cytokines in astrocytes [43] and microglia [42], and has the potential to participate in several brain diseases including AD [42]. Consistent with the reported tryptophan metabolites in human CSF [16], our findings added evidence that fecal tryptophan metabolism might be under the control of microbiota and the crosstalk of microbiota-host might play a role in the pathogenesis of $\mathrm{AD}$.

Interestingly, we first revealed that fecal IPYA was enriched in pre-onset aMCI and $\mathrm{AD}$, and significantly linked with intestinal dysbiosis and $\mathrm{AD}$ development. Notably, fecal IPYA could classify AD and aMCI from normal cognitive HC. IPYA is one of the major precursor metabolites that can be converted into numerous AhR ligands [42]. However, we did not find coordinately enriched AhR ligands in the intestine of AD patients, suggesting that the metabolic conversion of IPYA to AhR ligands was suppressed in aMCI and AD. Although AhR signaling gained has considerable attention in the field of $A D$, there have been no reports of dysregulation of intestinal IPYA in AD. Taken together, our results demonstrate that fecal tryptophan perturbation in AD, especially IPYA, which might be the mediator of intestinal dysbiosis and participate in $\mathrm{AD}$ development, is therefore a potential target for preventing or treating $\mathrm{AD}$ from a therapeutic perspective.

Notably, we found that SCFAs had strong predictive ability for the conversion from aMCI to AD. SCFAs are known to beneficially maintain both intestinal barrier function $[8,44]$, and protect the integrity of the blood-brain barrier (BBB) [45,46]. Importantly, SCFAs directly influence the immune response [47], cross the BBB, and drive the maturation and function of microglia cells [48-50], which are the main macrophages in brain parenchyma. Moreover, SCFAs exert their effects by regulating the secretion of gut hormones like glucagonlike peptide-1, and these hormones could improve neuroplasticity in the hippocampus [8]. Interestingly, the restoration of the intestinal SCFAs help prevent or ameliorate AD pathology [51]. More recently, a study reported that the modified Mediterranean-ketogenic diet modulated the IM and improved SCFAs in MCI patients associated with the improved AD biomarkers in CSF [48]. Thus, the decreased SCFAs in intestine might be crucial mediators between IM and AD.

In addition, we first found that the fecal LCA decreased in AD and pre-onset aMCI were correlated with cognitive impairment. As the most hydrophobic BAs, LCA was the secondary BA and could cross the BBB to exert a cytotoxic effect for the brain $[13,33]$. Since LCA is converted from CDCA via $7 \alpha$-dehydroxylation by IM, the unchanged CDCA and ratio of LCA: CDCA in AD indicated the enhanced re-absorption of LCA into the circulating system and potentially impaired the CNS. In line with this finding, the increased LCA in AD was reported in both the serum of patients [52,53] and the brain tissue of AD transgenic mice [54], but was not found in other reported studies [13]. These inconsistent findings might be due to the different sample types and the uncontrolled effects of factors among cohorts from different geographical origins of western and eastern countries. Taken together, our findings add new clues for the role of gut-derived bile acid LCA in the pathogenesis of $\mathrm{AD}$. This deserved further study in a larger cohort.

We hypothesized that the gut dysbiosis associated with $\mathrm{AD}$ patients resulted in the dysregulation metabolism of tryptophan, SCFAs, and BAs. Furthermore, the perturbation of intestinal microbiota-metabolites could increase intestinal permeability, permit LPS 
translocation, or directly incur or aggravate neuroinflammation and neurotransmission disorders, and finally promote $\mathrm{AD}$ progression.

Despite these exciting findings of $\mathrm{AD}$, there were some limitations in this study. For instance, the diagnosis of AD was made according to the criteria of DSM-IV and guidelines of NINCDS-ADRDA [10]. Nevertheless, these criteria have been widely used for population-based studies and reported with quite high accuracy by the postmortem analysis [19]. Here, the recruited volunteers refused to perform the lumbar puncture for the CSF assay and positron emission tomography imaging, which have a low degree of acceptance in the elderly [55]. Second, this observational study failed to demonstrate the causality role of microbial metabolites in AD development. However, most of these metabolites were progressively altered with disease progression and significantly correlated with cognitive impairment, strongly indicating their critical role in AD. Further longitudinal studies in large cohorts are required to focus on more detailed periods and follow-up investigation to define their cause-effect relationship.

\section{Conclusions}

In the present study, the highly reproducible and high-throughput targeted and untargeted UPLC/GC-MS were used to profile the fecal metabolomics of pre-onset aMCI and $\mathrm{AD}$, and revealed the altered fecal microbial signatures for $\mathrm{AD}$. The perturbation of fecal microbiota-metabolomics pointed to the dysregulation pathways of tryptophan, SCFAs and bile acids. Intriguingly, fecal IPYA was first reported to discriminate both preonset $\mathrm{aMCI}$ and $\mathrm{AD}$ from $\mathrm{HC}$, and five SCFAs discriminated $\mathrm{AD}$ from aMCI. Importantly, the best results for detecting $\mathrm{AD}$ and aMCI were achieved when IPYA was combined with five SCFA measurements. Thus, these findings provide the evidence that fecal microbial signatures could be noninvasive biomarkers for $\mathrm{AD}$ screening and management, and targeting IM or microbial metabolism might be a promising method for preventing and treating AD.

Supplementary Materials: The following are available online at https:/ /www.mdpi.com/2072-6 643/13/1/228/s1 including Supplementary Materials and Methods, Supplementary Tables S1-S9, Supplementary Figures S1-S6.

Author Contributions: Conceptualization, B.W.; Data curation, L.W., Y.H., L.L. (Liang Li), and B.W.; Funding acquisition, L.L. (Lanjuan Li) and B.W.; Methodology, L.W., Y.H., Z.Z., G.P., P.L., S.Y., S.Z., J.C., H.L., L.S., Y.S., Y.W., and B.W.; Project administration, B.W.; Writing-original draft, L.W. and B.W.; Writing - review \& editing, B.W. and L.L. (Lanjuan Li) All authors have read and agreed to the published version of the manuscript.

Funding: This study was funded by National Natural Science Foundation of China (grant numbers 81790633, 81790630, 30901190); the CAMS Innovation Fund for Medical Sciences (grant numbers 2019I2M-5-045); and the Zhejiang Provincial Health Technology Plan (grant numbers No. 2010KYA077).

Institutional Review Board Statement: The study was conducted according to the guidelines of the Declaration of Helsinki, and approved by the Ethics Committee of the First Affiliated Hospital, College of Medicine, Zhejiang University (2019-251).

Informed Consent Statement: Informed consent was obtained from all subjects involved in the study.

Data Availability Statement: The sequence dataset of the fecal microbiota can be downloaded from the National Center for Biotechnology Information (NCBI) Bioproject database (PRJNA496408).

Acknowledgments: We thank the healthy individuals, patients, and their spouses who donated their samples, the clinical doctors who provided help in the sample collection, and Men Yu from the Shanghai Metabolome Institute-Wuhan (SMI) for technique assistance in the quantity detection of SCFAs and bile acids.

Conflicts of Interest: The authors declare no conflict of interest. 


\section{References}

1. Sperling, R.A.; Aisen, P.S.; Beckett, L.A.; Bennett, D.A.; Craft, S.; Fagan, A.M.; Iwatsubo, T.; Jack, C.R., Jr.; Kaye, J.; Montine, T.J.; et al. Toward defining the preclinical stages of Alzheimer's disease: Recommendations from the National Institute on Aging-Alzheimer's Association workgroups on diagnostic guidelines for Alzheimer's disease. Alzheimer's Dement. J. Alzheimer's Assoc. 2011, 7, 280-292. [CrossRef] [PubMed]

2. Australia, D.; Baker, S.; Banerjee, S. Alzheimer's Disease International. World Alzheimer Report 2019: Attitudes to Dementia; Alzheimer's Disease International: London, UK, 2019.

3. Alzheimer's Association. 2019 Alzheimer's disease facts and figures. Alzheimer's Dement. 2019, 15, 321-387. [CrossRef]

4. Levey, A.I.; Lah, J.; Goldstein, F.; Steenland, K.; Bliwise, D. Mild cognitive impairment: An opportunity to identify patients at high risk for progression to Alzheimer's disease. Clin. Ther. 2006, 28, 991-1001. [CrossRef] [PubMed]

5. Cortes-Canteli, M.; Iadecola, C. Alzheimer's Disease and Vascular Aging: JACC Focus Seminar. J. Am. Coll. Cardiol. 2020, 75, 942-951. [CrossRef]

6. Busche, M.A.; Hyman, B.T. Synergy between amyloid- $\beta$ and tau in Alzheimer's disease. Nat. Neurosci. 2020, $23,1183-1193$. [CrossRef]

7. Agus, A.; Planchais, J.; Sokol, H. Gut Microbiota Regulation of Tryptophan Metabolism in Health and Disease. Cell Host Microbe 2018, 23, 716-724. [CrossRef]

8. Dalile, B.; Van Oudenhove, L.; Vervliet, B.; Verbeke, K. The role of short-chain fatty acids in microbiota-gut-brain communication. Nat. Rev. Gastroenterol. Hepatol. 2019, 16, 461-478. [CrossRef]

9. Vogt, N.M.; Kerby, R.L.; Dill-McFarland, K.A. Gut microbiome alterations in Alzheimer's disease. Sci. Rep. $2017,7,13537$. [CrossRef]

10. Liu, P.; Wu, L.; Peng, G.; Han, Y.; Tang, R.; Ge, J.; Zhang, L.; Jia, L.; Yue, S.; Zhou, K.; et al. Altered microbiomes distinguish Alzheimer's disease from amnestic mild cognitive impairment and health in a Chinese cohort. Brain Behav. Immun. 2019, 80, 633-643. [CrossRef]

11. Harach, T.; Marungruang, N.; Duthilleul, N.; Cheatham, V.; Mc Coy, K.D.; Frisoni, G.; Neher, J.; Fåk, F.; Jucker, M.; Lasser, T.; et al. Reduction of Abeta amyloid pathology in APPPS1 transgenic mice in the absence of gut microbiota. Sci. Rep. 2017, 7, 41802. [CrossRef]

12. Zierer, J.; Jackson, M.A. The fecal metabolome as a functional readout of the gut microbiome. Nat. Genet. 2018, 50, 790-795. [CrossRef]

13. Mahmoudiandehkordi, S.; Arnold, M.; Nho, K.; Ahmad, S.; Jia, W.; Xie, G.; Louie, G.; Kueider-Paisley, A.; Moseley, M.A.; Thompson, J.W.; et al. Altered bile acid profile associates with cognitive impairment in Alzheimer's disease-An emerging role for gut microbiome. Alzheimer's Dement. J. Alzheimer's Assoc. 2019, 15, 76-92. [CrossRef] [PubMed]

14. Cui, Y.; Liu, X.; Wang, M.; Liu, L.; Sun, X.; Ma, L.; Xie, W.; Wang, C.; Tang, S.; Wang, D.; et al. Lysophosphatidylcholine and amide as metabolites for detecting alzheimer disease using ultrahigh-performance liquid chromatography-quadrupole time-of-flight mass spectrometry-based metabonomics. J. Neuropathol. Exp. Neurol. 2014, 73, 954-963. [CrossRef]

15. Varma, V.R.; Oommen, A.; Varma, S.; Casanova, R.; An, Y.; Andrews, R.M.; O’Brien, R.; Pletnikova, O.; Troncoso, J.C.; Toledo, J.B.; et al. Brain and blood metabolite signatures of pathology and progression in Alzheimer disease: A targeted metabolomics study. PLoS Med. 2018, 15, e1002482. [CrossRef]

16. Kaddurah-Daouk, R.; Rozen, S.; Matson, W.; Han, X.; Hulette, C.M.; Burke, J.R.; Doraiswamy, P.M.; Welsh-Bohmer, K.A. Metabolomic changes in autopsy-confirmed Alzheimer's disease. Alzheimer's Dement. J. Alzheimer's Assoc. 2011, 7, $309-317$. [CrossRef]

17. Diagnostic and Statistical Manual of Mental Disorders, 4th ed.; American Psychiatric Association: Washington, DC, USA, $1994 ;$ p. 886.

18. Dubois, B.; Feldman, H.H.; Jacova, C.; DeKosky, S.T.; Barberger-Gateau, P.; Cummings, J.L.; Delacourte, A.; Galasko, D.; Gauthier, S.; Jicha, G.A.; et al. Research criteria for the diagnosis of Alzheimer's disease: Revising the NINCDS-ADRDA criteria. Lancet Neurol. 2007, 6, 734-746. [CrossRef]

19. Petersen, R.C. Mild cognitive impairment as a diagnostic entity. J. Intern. Med. 2004, 256, 183-194. [CrossRef] [PubMed]

20. Li, M.; Wang, B.; Zhang, M.; Rantalainen, M.; Wang, S.; Zhou, H.; Zhang, Y.; Shen, J.; Pang, X.; Zhang, M.; et al. Symbiotic gut microbes modulate human metabolic phenotypes. Proc. Natl. Acad. Sci. USA 2008, 105, 2117-2122. [CrossRef]

21. Wang, B.; Chen, D.; Chen, Y.; Hu, Z.; Cao, M.; Xie, Q.; Chen, Y.; Xu, J.; Zheng, S.; Li, L. Metabonomic Profiles Discriminate Hepatocellular Carcinoma from Liver Cirrhosis by Ultraperformance Liquid Chromatography-Mass Spectrometry. J. Proteome Res. 2012, 11, 1217-1227. [CrossRef]

22. Cao, H.; Huang, H.; Xu, W.; Chen, D.; Yu, J.; Li, J.; Li, L. Fecal metabolome profiling of liver cirrhosis and hepatocellular carcinoma patients by ultra performance liquid chromatography-mass spectrometry. Anal. Chim. Acta 2011, 691, 68-75. [CrossRef] [PubMed]

23. Simonato, M.; Fochi, I.; Vedovelli, L.; Giambelluca, S.; Carollo, C.; Padalino, M.; Carnielli, V.P.; Cogo, P. Urinary metabolomics reveals kynurenine pathway perturbation in newborns with transposition of great arteries after surgical repair. Metab. Off. J. Metab. Soc. 2019, 15, 145. [CrossRef] [PubMed]

24. Chen, J.; Wang, W.; Lv, S.; Yin, P.; Zhao, X.; Lu, X.; Zhang, F.; Xu, G. Metabonomics study of liver cancer based on ultra performance liquid chromatography coupled to mass spectrometry with HILIC and RPLC separations. Anal. Chim. Acta 2009, 650, 3-9. [CrossRef] [PubMed] 
25. Furuhashi, T.; Sugitate, K.; Nakai, T.; Jikumaru, Y.; Ishihara, G. Rapid profiling method for mammalian feces short chain fatty acids by GC-MS. Anal. Biochem. 2018, 543, 51-54. [CrossRef]

26. Zheng, X.; Qiu, Y.; Zhong, W.; Baxter, S.; Su, M.; Li, Q.; Xie, G.; Ore, B.M.; Qiao, S.; Spencer, M.D.; et al. A targeted metabolomic protocol for short-chain fatty acids and branched-chain amino acids. Metab. Off. J. Metab. Soc. 2013, 9, 818-827. [CrossRef] [PubMed]

27. Tang, T.W.; Chen, H.-C.; Chen, C.-Y.; Yen, C.Y.T.; Lin, C.-J.; Prajnamitra, R.P.; Chen, L.-L.; Ruan, S.-C.; Lin, J.-H.; Lin, P.-J.; et al. Loss of Gut Microbiota Alters Immune System Composition and Cripples Postinfarction Cardiac Repair. Circulation 2019, 139, 647-659. [CrossRef] [PubMed]

28. Lin, H.; An, Y.; Tang, H.; Wang, Y. Alterations of Bile Acids and Gut Microbiota in Obesity Induced by High Fat Diet in Rat Model. J. Agric. Food Chem. 2019, 67, 3624-3632. [CrossRef]

29. Penno, C.A.; Arsenijevic, D.; Da Cunha, T.; Kullak-Ublick, G.A.; Montani, J.-P.; Odermatt, A. Quantification of multiple bile acids in uninephrectomized rats using ultra-performance liquid chromatography-tandem mass spectrometry. Anal. Methods 2013, 5, 1155-1164. [CrossRef]

30. Zhou, S.Y.; Gillilland, M., 3rd; Wu, X.; Leelasinjaroen, P.; Zhang, G.; Zhou, H.; Ye, B.; Lu, Y.; Owyang, C. FODMAP diet modulates visceral nociception by lipopolysaccharide-mediated intestinal inflammation and barrier dysfunction. J. Clin. Investig. 2018, 128, 267-280. [CrossRef]

31. De Hoon, M.J.L.; Imoto, S.; Nolan, J.; Miyano, S. Open source clustering software. Bioinformatics 2004, 20, 1453-1454. [CrossRef]

32. Ma, Y.; Li, F.; Wang, L.; Zhao, W.; Li, D.; Xian, Y.; Jiang, X. A risk prediction model of sperm retrieval failure with fine needle aspiration in males with non-obstructive azoospermia. Hum. Reprod. 2019, 34, 200-208. [CrossRef]

33. Tripodi, V.; Contin, M.; Fernández, M.A.; Lemberg, A. Bile acids content in brain of common duct ligated rats. Ann. Hepatol. 2012, 11,930-934. [CrossRef]

34. Van Dyke, R.W.; Stephens, J.E.; Scharschmidt, B.F. Bile acid transport in cultured rat hepatocytes. Am. J. Physiol. 1982, 243, G484-G492. [CrossRef] [PubMed]

35. Krähenbühl, S.; Talos, C.; Fischer, S.; Reichen, J. Toxicity of bile acids on the electron transport chain of isolated rat liver mitochondria. Hepatology 1994, 19, 471-479. [CrossRef] [PubMed]

36. Zeng, H.; Chi, H. Metabolic control of regulatory T cell development and function. Trends Immunol. 2015, 36, 3-12. [CrossRef]

37. Alkasir, R.; Li, J.; Li, X.; Jin, M.; Zhu, B. Human gut microbiota: The links with dementia development. Protein Cell 2017, 8, 90-102. [CrossRef]

38. Koh, A.; De Vadder, F.; Kovatcheva-Datchary, P.; Bäckhed, F. From Dietary Fiber to Host Physiology: Short-Chain Fatty Acids as Key Bacterial Metabolites. Cell 2016, 165, 1332-1345. [CrossRef]

39. Sparkman, N.L.; Buchanan, J.B.; Heyen, J.R.R.; Chen, J.; Beverly, J.L.; Johnson, R.W. Interleukin-6 facilitates lipopolysaccharideinduced disruption in working memory and expression of other proinflammatory cytokines in hippocampal neuronal cell layers. J. Neurosci. Off. J. Soc. Neurosci. 2006, 26, 10709-10716. [CrossRef]

40. de J.R. De-Paula, V.; Forlenza, A.S.; Forlenza, O.V. Relevance of gutmicrobiota in cognition, behaviour and Alzheimer's disease. Pharmacol. Res. 2018, 136, 29-34. [CrossRef]

41. Xu, Y.; Yan, J.; Zhou, P.; Li, J.; Gao, H.; Xia, Y.; Wang, Q. Neurotransmitter receptors and cognitive dysfunction in Alzheimer's disease and Parkinson's disease. Prog. Neurobiol. 2012, 97, 1-13. [CrossRef]

42. Ma, N.; He, T.; Johnston, L.J.; Ma, X. Host-microbiome interactions: The aryl hydrocarbon receptor as a critical node in tryptophan metabolites to brain signaling. Gut Microbes 2020, 11, 1203-1219. [CrossRef]

43. Rothhammer, V.; Mascanfroni, I.D.; Bunse, L.; Takenaka, M.C.; Kenison, J.E.; Mayo, L.; Chao, C.-C.; Patel, B.; Yan, R.; Blain, M.; et al. Type I interferons and microbial metabolites of tryptophan modulate astrocyte activity and central nervous system inflammation via the aryl hydrocarbon receptor. Nat. Med. 2016, 22, 586-597. [CrossRef] [PubMed]

44. Rooks, M.G.; Garrett, W.S. Gut microbiota, metabolites and host immunity. Nat. Rev. Immunol. 2016, 16, 341-352. [CrossRef] [PubMed]

45. Braniste, V.; Al-Asmakh, M.; Kowal, C.; Anuar, F.; Abbaspour, A.; Tóth, M.; Korecka, A.; Bakocevic, N.; Ng, L.G.; Kundu, P.; et al. The gut microbiota influences blood-brain barrier permeability in mice. Sci. Transl. Med. 2014, 6, 263ra158. [CrossRef] [PubMed]

46. Hoyles, L.; Snelling, T.; Umlai, U.-K.; Nicholson, J.K.; Carding, S.R.; Glen, R.C.; McArthur, S. Microbiome-host systems interactions: Protective effects of propionate upon the blood-brain barrier. Microbiome 2018, 6, 55. [CrossRef] [PubMed]

47. Huuskonen, J.; Suuronen, T.; Nuutinen, T.; Kyrylenko, S.; Salminen, A. Regulation of microglial inflammatory response by sodium butyrate and short-chain fatty acids. Br. J. Pharmacol. 2004, 141, 874-880. [CrossRef]

48. Nagpal, R.; Neth, B.J.; Wang, S.; Craft, S.; Yadav, H. Modified Mediterranean-ketogenic diet modulates gut microbiome and short-chain fatty acids in association with Alzheimer's disease markers in subjects with mild cognitive impairment. EBioMedicine 2019, 47, 529-542. [CrossRef]

49. Silva, Y.P.; Bernardi, A.; Frozza, R.L. The Role of Short-Chain Fatty Acids From Gut Microbiota in Gut-Brain Communication. Front. Endocrinol. 2020, 11, 25. [CrossRef]

50. Erny, D.; De Angelis, A.L.H.; Jaitin, D.; Wieghofer, P.; Staszewski, O.; David, E.; Keren-Shaul, H.; Mahlakoiv, T.; Jakobshagen, K.; Buch, T.; et al. Host microbiota constantly control maturation and function of microglia in the CNS. Nat. Neurosci. 2015, 18, 965-977. [CrossRef] 
51. Govindarajan, N.; Agis-Balboa, R.C.; Walter, J.; Sananbenesi, F.; Fischer, A. Sodium butyrate improves memory function in an Alzheimer's disease mouse model when administered at an advanced stage of disease progression. J. Alzheimer's Dis. JAD 2011, 26, 187-197. [CrossRef] [PubMed]

52. Olazarán, J.; Gil-De-Gómez, L.; Rodríguez-Martín, A.; Valentí-Soler, M.; Frades-Payo, B.; Marín-Muñoz, J.; Antúnez, C.; FrankGarcía, A.; Acedo-Jiménez, C.; Morlán-Gracia, L.; et al. A blood-based, 7-metabolite signature for the early diagnosis of Alzheimer's disease. J. Alzheimer's Dis. JAD 2015, 45, 1157-1173.

53. Marksteiner, J.; Blasko, I.; Kemmler, G.; Koal, T.; Humpel, C. Bile acid quantification of 20 plasma metabolites identifies lithocholic acid as a putative biomarker in Alzheimer's disease. Metab. Off. J. Metab. Soc. 2018, 14, 1. [CrossRef] [PubMed]

54. Pan, X.; Elliott, C.T.; McGuinness, B.; Passmore, P.; Kehoe, P.G.; Holscher, C.; McClean, P.L.; Graham, S.F.; Green, B.D. Metabolomic Profiling of Bile Acids in Clinical and Experimental Samples of Alzheimer's Disease. Metabolites 2017, 7, 28. [CrossRef] [PubMed]

55. Cui, M.; Jiang, Y.; Zhao, Q.; Zhu, Z.; Liang, X.; Zhang, K.; Wu, W.; Dong, Q.; An, Y.; Tang, H.; et al. Metabolomics and incident dementia in older Chinese adults: The Shanghai Aging Study. Alzheimer's Dement. J. Alzheimer's Assoc. 2020, 16, 779-788. [CrossRef] [PubMed] 